\title{
Consumo de antibióticos em um hospital de alta complexidade: padrão de utilização em diferentes enfermarias
}

Antibiotic utilization in a high complexity hospital: pattern of use in different wards

Uso de antibióticos en un hospital de alta complejidad: patrón de uso en diferentes enfermerías

Recebido: 10/01/2022 | Revisado: 14/01/2022 | Aceito: 18/01/2022 | Publicado: 190/01/2022

Lucas Borges Pereira

ORCID: https://orcid.org/0000-0002-5957-9286 Universidade de São Paulo, Brasil

E-mail: lucasborgespereira82@gmail.com

Maria Olívia Barboza Zanetti

ORCID: https://orcid.org/0000-0002-9551-4711

Universidade de São Paulo, Brasil

E-mail: marolzanetti@gmail.com

João Paulo Vilela Rodrigues

ORCID: https://orcid.org/0000-0002-5785-1418

Universidade de São Paulo, Brasil

E-mail: jpvilela@fcfrp.usp.br

Leonardo Régis Leira Pereira

ORCID: https://orcid.org/0000-0002-8609-1390

Universidade de São Paulo, Brasil

E-mail: 1pereira@fcfrp.usp.br

\begin{abstract}
Resumo
Objetivo: Avaliar o padrão de utilização de antibióticos entre as diferentes enfermarias de um hospital universitário de alta complexidade. Métodos: Estudo descritivo realizado no Hospital das Clínicas da Faculdade de Medicina de Ribeirão Preto. Buscou-se no prontuário e prescrição eletrônicos dados clínicos e de medicamentos dos pacientes internados, com 18 anos ou mais, que receberam prescrição de antibiótico no ano de 2014. As prescrições de antibióticos foram classificadas em tratamento empírico (TE) e tratamento direcionado (TD), e seu consumo foi calculado por meio da dose diária definida por 1.000 pacientes-dia, e apresentado por meio do método de Utilização de Medicamentos 90\%. Resultados: 4.102 indivíduos tiveram prescrições de antibióticos com finalidade terapêutica. $63.985(84,6 \%)$ prescrições foram classificadas como TE, e $11.660(15,4 \%)$ prescrições foram classificadas como TD. As cefalosporinas de $4^{a}$ geração e os glicopeptídeos estão entre aqueles que representaram $90 \%$ do consumo em todas as enfermarias. No grupo TD, antibióticos de amplo espectro de ação mantiveram elevado consumo nas unidades, como os carbapenêmicos que foram os mais utilizados em três unidades, e a polimixina mais utilizada na unidade de terapia intensiva. Por outro lado, as penicilinas resistentes à betalactamase passaram a ser representadas em um número maior de enfermarias em relação à TE. Conclusão: O perfil de consumo de antibióticos sugere a hipótese de que os exames de antibiograma estão sendo realizados com o objetivo de investigar o perfil de sensibilidade bacteriana quando há falha terapêutica, e não para descalonar o tratamento.
\end{abstract}

Palavras-chave: Uso de medicamentos; Antibacterianos; Resistência microbiana a medicamentos; Uso indevido de medicamentos; Gestão de antimicrobianos; Beta-lactâmicos.

\footnotetext{
Abstract

Objective: To evaluate the pattern of antibiotic use among different wards of a high-complexity university hospital. Methods: Descriptive study conducted at the Hospital das Clínicas da Faculdade de Medicina de Ribeirão Preto. Clinical and drugs utilization data of inpatients, aged 18 years or older, who received antibiotic prescriptions in 2014 were collected in the medical record and electronic prescription. Antibiotic prescriptions were classified into empirical treatment (ET) and targeted treatment (DT), and their consumption was calculated using the defined daily dose per 1,000 patient-days. The presentation of antibiotic consumption was performed using the method Drug Utilization 90\%. Results: 4,102 individuals were prescribed antibiotics for therapeutic purposes. 63,985 (84.6\%) of the prescriptions were classified as ET, and 11,660 (15.4\%) prescriptions were classified as DT. 4th generation cephalosporins and glycopeptides are among those that accounted for $90 \%$ of utilization in all wards. In the DT group, antibiotics with a broad spectrum of action maintain high consumption in the units, such as carbapenems, the most used in three units, and polymyxin the most used in the intensive care unit. On the other hand, beta-lactamase resistant penicillins started to be represented in a greater number of wards compared to ET. Conclusion: The antibiotic
} 
utilization pattern suggests the hypothesis that antibiogram tests are being performed with the aim of investigating the bacterial sensitivity profile when there is therapeutic failure, and not to de-escalate treatment.

Keywords: Drug utilization; Anti-bacterial agents; Drug resistance, microbial; Prescription drug misuse; Antimicrobial stewardship; Beta-lactams.

\section{Resumen}

Objetivo: Evaluar el patrón de uso de antibióticos entre diferentes enfermerías de un hospital universitario de alta complejidad. Métodos: estudio descriptivo realizado en el Hospital das Clínicas da Facudade de Medicina de Ribeirão Preto. Se buscaron en la historia clínica y prescripción electrónica los datos clínicos y de medicación de los pacientes hospitalizados, de 18 o más años, que recibieron prescripción de antibióticos en 2014. Las prescripciones de antibióticos se clasificaron en tratamiento empírico (ET) y tratamiento dirigido (DT), y su consumo fue calculada utilizando la dosis diaria definida por 1.000 días-paciente, y presentada utilizando el método de utilización del fármaco al 90\%. Resultados: Se prescribieron antibióticos a 4.102 personas con fines terapéuticos. 63.985 (84,6\%) prescripciones se clasificaron como TE y $11.660(15,4 \%)$ prescripciones se clasificaron como DT. Las cefalosporinas de cuarta generación y glicopéptidos se encuentran entre los que representaron el $90 \%$ del consumo en todas las enfermerías. En el grupo DT, los antibióticos de amplio espectro mantuvieron un alto consumo en las unidades, como los carbapenémicos, que fueron los más utilizados en tres unidades, y la polimixina más utilizada en la unidad de cuidados intensivos. Por otro lado, las penicilinas resistentes a betalactamasas comenzaron a estar representadas en un mayor número de salas en comparación con ET. Conclusión: El perfil de consumo de antibióticos sugiere la hipótesis de que las pruebas de antibiograma se están realizando con el objetivo de investigar el perfil de sensibilidad bacteriana cuando hay fracaso terapéutico y no desescalar el tratamiento.

Palabras clave: Utilización de medicamentos; Antibacterianos; Farmacorresistencia microbiana; Abuso de medicamentos; Programas de optimización del uso de los antimicrobianos; Beta-lactamas.

\section{Introdução}

Os estudos de utilização de medicamentos compõem uma importante vertente da farmacoepidemiologia, uma vez que permitem investigar a comercialização, a distribuição, a prescrição e o uso de medicamentos em uma determinada população, gerando informações com potencial para transformar a realidade clínica, social ou econômica observada (Castro et al., 2002).

Os antimicrobianos tiveram uma importância inquestionável nas últimas décadas para a redução da mortalidade relacionada a doenças infecciosas. Embora qualquer uso de antibióticos, mesmo quando corretamente indicado, tenha relação com a seleção de bactérias resistentes, o uso inadequado na medicina, na agricultura e na veterinária correlaciona-se com o crescimento exponencial da resistência bacteriana (Kuster et al., 2008). O surto de inefetividade terapêutica destes fármacos, associado às tentativas falhas no desenvolvimento de novos antimicrobianos, representa um sinal alarmante para a saúde humana e animal, resultando em aumento da morbidade, mortalidade e custo dos cuidados de saúde (Kuster et al., 2008; Cheng et al. 2012).

Em geral, a prevalência de resistência bacteriana é mais alta no ambiente hospitalar do que na comunidade, dado que os pacientes hospitalizados necessitam mais frequentemente de prescrições de antibióticos. O tipo, o tamanho, a complexidade e a localização geográfica do hospital também podem influenciar o uso de antibióticos. A escolha inadequada do prescritor e a duração prolongada do tratamento, além da não conformidade com as precauções de controle de infecção hospitalar por parte dos profissionais de saúde, contribuem para este cenário (Benko et al., 2009).

Estudos de utilização de antibióticos que avaliem padrões e tendências podem ser úteis para a otimização do uso hospitalar desta classe de fármacos e contribuir para a redução da resistência bacteriana. Diante disto, o objetivo do presente estudo foi avaliar o padrão de utilização de antibióticos entre as diferentes enfermarias de um hospital universitário de alta complexidade.

\section{Metodologia}

\section{Local do estudo}

Este foi um estudo descritivo realizado no Hospital das Clínicas da Faculdade de Medicina de Ribeirão Preto 
(HCFMRP), hospital universitário de 744 leitos, localizado no município de Ribeirão Preto, estado de São Paulo, Brasil. Esta instituição oferece um serviço de cuidado à saúde de nível terciário, que faz parte do sistema público de saúde brasileiro, o Sistema Único de Saúde (SUS) (Medronho et al., 2008).

O HCFMRP possui sistemas eletrônicos de informação, como o Sistema de Apoio à Atenção Hospitalar (ATHOS) e a Prescrição Eletrônica, cuja função é aprimorar a gestão de informações sociodemográficas, clínicas e farmacoterapêuticas dos pacientes. Sendo assim, as informações sobre os pacientes são armazenadas em um banco de dados no hospital.

\section{Coleta de dados e população do estudo}

O sistema informatizado do hospital foi utilizado como fonte de informação sobre a utilização de antibióticos pelos pacientes. Buscou-se no banco de dados todas as prescrições realizadas entre 01 de janeiro de 2014 à 31 de dezembro de 2014 , que apresentavam pelo menos um antimicrobiano pertencente a classificação J01 (antibacterianos para uso sistêmico) do sistema de classificação farmacológica Anatomical Therapeutic Chemical (ATC), versão 2015, elaborado pela Organização Mundial de Saúde (OMS) (WHO, 2015). Foram excluídas todas as prescrições realizadas para pessoas menores de 18 anos ou que tivessem a finalidade de profilaxia.

A partir destas prescrições, obteve-se as seguintes informações a respeito do paciente para o qual o antibiótico foi indicado: idade, sexo, dados clínicos do período de internação (tempo de internação e diagnóstico que motivou a internação, conforme a Classificação Estatística Internacional de Doenças e Problemas Relacionados com a Saúde, versão 2014) (WHO, 2014). Sobre a antibioticoterapia, coletou-se nome do medicamento (nome do princípio ativo), dose diária, tempo de tratamento, se uso profilático ou uso terapêutico, se tratamento empírico (TE) ou tratamento direcionado (TD). Por último, foram coletados dados sobre os exames de identificação da bactéria causadora da infecção e seu perfil de sensibilidade para os antibióticos. Estes exames são processados pelo setor de microbiologia do HCFMRP que segue os mesmos procedimentos padronizados pelo Clinical and Laboratory Standards Institute (CLSI).

\section{Análise dos dados}

A caracterização da amostra incluída no estudo foi feita por meio do cálculo da média, desvio padrão, frequências absoluta e relativa.

Os pacientes incluídos no estudo foram divididos em dois grupos: aqueles que receberam antibióticos para TE, e aqueles que receberam para TD. No entanto, houve casos em que o tratamento do paciente foi ajustado após resultado de cultura bacteriana. Estes indivíduos foram incluídos nos dois grupos de acordo com o período durante o qual foi submetido a cada uma das terapias (TE ou TD). Dessa maneira, em relação aos dados de tempo de internação e diagnóstico clínico principal, calculou-se, para os dois grupos, média e desvio padrão para a primeira variável, e frequências absoluta e relativa para a variável diagnóstico clínico principal. O tempo de internação do indivíduo que foi submetido ao TE e ao TD foi incluído no cálculo da média de cada grupo.

Alguns pacientes foram internados em mais de uma ocasião durante o período do estudo. Os dados de cada nova admissão hospitalar foram contabilizados como indivíduos diferentes no estudo.

O sistema de classificação e métrica de consumo de fármacos ATC/dose diária definida (DDD) é uma ferramenta recomendada pela OMS para apresentar os resultados de estudos de utilização de medicamentos e que permite realizar comparações entre diferentes estudos. Sendo assim, os resultados de consumo de antibióticos foram apresentados levando em consideração o quarto nível da classificação ATC (WHO, 2015). Foi utilizada a versão de 2015.

O consumo de cada antibiótico foi calculado por meio da Dose Diária Definida por 1.000 pacientes-dia (DDD/1.000 pacientes-dia) para cada enfermaria por meio da equação abaixo (WHO, 2015): 


\section{$\mathrm{DDD} / 1.000$}

pacientes-dia
$=$ Total de antibiótico utilizado em gramas

DDD do antibiótico $\times$ pacientes-dia da enfermaria no ano de 2014

A apresentação dos resultados de consumo de antibióticos foi realizada utilizando o método de Utilização de Medicamentos 90\% (do inglês, Drug Utilization 90\%, DU90\%) para cada enfermaria. Este método apresenta o consumo dos grupos de antibióticos que representa 90\% do consumo total (Bergman et al., 1998).

Os dados de identificação das bactérias e seu perfil de sensibilidade em relação aos antimicrobianos foram analisados utilizando frequência relativa. O perfil de sensibilidade das bactérias aos antibióticos foi apresentado como sensível, intermediário e resistente.

\section{Aprovação ética}

Este estudo foi aprovado ad referendum pelos Comitês de Ética da Faculdade de Ciências Farmacêuticas de Ribeirão Preto (protocolo 1.124.047 de 25 Junho de 2015) e do HCFMRP (protocolo 1.139.544 de 25 Junho de 2015).

\section{Resultados}

Foram encontradas 90.475 prescrições de antimicrobianos referentes a 7.287 pacientes. Dentre estes, 43,7\% (3.185) foram excluídos do estudo por tratar-se de prescrições profiláticas. Assim, as prescrições de 4.102 indivíduos foram estudadas ( $\mathrm{n}=75.645$ ), com média de idade de 54,0 anos (DP 15,6) e prevalência do sexo feminino $(50,9 \%, 2.087$ ).

Sobre a análise do tipo de tratamento proposto, para 96,7\% (3.965) dos pacientes foi prescrito pelo menos um antibiótico para TE que representaram 84,6\% (63.985) das prescrições. Por sua vez, aqueles que receberam pelo menos uma prescrição de antibiótico para TD corresponderam a 20,6\% (847) dos pacientes e 15,4\% (11.660) das prescrições. No grupo que recebeu TE havia 48,4\% de mulheres e a média de idade foi de 52,2 (DP 17,5) anos, enquanto no grupo que recebeu TD, $53,6 \%$ eram mulheres e a média de idade foi de 52,5 (DP 18,0) anos.

No grupo TE houve 5.106 internações, das quais 9,9\% duraram mais do que 30 dias. A média do tempo de internação neste grupo foi de 30,9 dias (DP 18,3). No grupo de TD houve 990 internações, das quais 27,8\% duraram mais do que 30 dias. A média do tempo de internação neste grupo foi de 48,9 dias (DP 28,9). Em ambos os grupos o principal motivo de internação foi neoplasias (TE: 29,4\%; TD: 23,5\%) seguido de doenças do sistema circulatório (TE: 12,9\%; TD: 19,4\%).

Em relação à utilização de antibióticos, $84,0 \%$ consistiram em TE. O perfil de utilização de antimicrobianos que correspondem aos $90 \%$ mais consumidos entre as enfermarias está representado na Tabela 1. A unidade de terapia intensiva (UTI) e a unidade especial de tratamento de doenças infecciosas (UETDI) foram as unidades que mais utilizaram antibióticos para TE de doenças infecciosas durante o período de estudo. 
Research, Society and Development, v. 11, n. 2, e12011225573, 2022

(CC BY 4.0) | ISSN 2525-3409 | DOI: http://dx.doi.org/10.33448/rsd-v11i2.25573

Tabela 1. Representação da Utilização de Antibióticos 90\% de acordo com as classes de antibióticos, classificadas pela ATC, para tratamento empírico em cada enfermaria.

\begin{tabular}{|c|c|c|c|c|c|c|c|c|c|c|c|c|c|c|}
\hline Classes de antibióticos/Enfermarias & UTI & UTR & TMO & $U C$ & UETDI & Ortop & Onco & MI & Oft_Otor & $C M 6^{\circ}$ & $C M 5^{\circ}$ & GO & Derm & Neuro \\
\hline Carbapenêmicos - J01DH & 550,82 & 112,41 & 134,85 & 125,28 & 105,82 & 30,09 & 80,04 & 89,44 & 51,98 & 107,88 & 37,39 & & 50,61 & $\overline{42,87}$ \\
\hline Antibacterianos glicopeptídicos - J01XA & 242,71 & 44,39 & 96,12 & 141,01 & 92,49 & 53,40 & 62,69 & 48,96 & 32,40 & 70,01 & 30,10 & 13,03 & 51,82 & 26,35 \\
\hline Polimixinas - J01XB & 197,41 & & & & & & & & & & & & & \\
\hline $\begin{array}{r}\text { Combinação de penicilina com inibidor de beta- } \\
\text { lactamase - J01CR }\end{array}$ & 148,11 & 44,62 & 21,91 & 51,32 & 84,24 & 23,73 & 68,41 & 91,52 & 62,95 & 68,53 & 46,16 & 11,14 & 43,17 & 29,11 \\
\hline Cefalosporinas de quarta geração - J01DE & 147,30 & 46,91 & 323,80 & 115,09 & 143,35 & 25,58 & 200,09 & 49,43 & 63,44 & 237,67 & 60,07 & 26,48 & 108,98 & 29,87 \\
\hline Combinação de sulfonamidas e trimetoprim - J01EE & 123,91 & 87,77 & 188,25 & & 347,68 & & & 130,76 & & 65,84 & & & 36,65 & 12,00 \\
\hline Outros aminoglicosídeos - J01GB & 105,95 & & & 23,10 & & 35,26 & & & & & 15,49 & 21,02 & & \\
\hline Cefalosporinas de terceira geração - J01DD & 70,12 & 97,72 & & 46,53 & 122,12 & 28,34 & 89,37 & 127,78 & 182,73 & 52,50 & 78,78 & 22,94 & 64,96 & 18,60 \\
\hline Tetraciclinas - J01AA & 58,64 & & & & & & & & & & & & 21,43 & \\
\hline Macrolídeos - J01FA & & 41,70 & & 21,20 & 189,35 & & 22,45 & 62,05 & & & 10,77 & & & \\
\hline Penicilinas resistentes a beta-lactamase - J01CF & & 46,22 & & & & 52,07 & & 117,44 & 201,77 & 56,00 & 45,94 & 31,84 & 121,39 & \\
\hline Fluoroquinolonas - J01MA & & 62,58 & 72,26 & 31,31 & 68,41 & 143,75 & 47,98 & 91,87 & 97,46 & 44,45 & 35,66 & 28,33 & 73,51 & 41,31 \\
\hline Penicilinas de amplo espectro - J01CA & & & & 25,06 & & & & & & & & 7,68 & & \\
\hline Lincosamidas - J01FF & & & & 21,84 & 108,76 & 46,90 & 29,86 & 36,78 & 135,41 & 35,94 & 14,95 & 30,92 & 35,53 & 12,70 \\
\hline Sulfonamidas de ação intermediária - J01EC & & & & & 415,89 & & & 189,60 & & & & & & \\
\hline Cefaloporinas de segunda geração - J01DC & & & & & & & & & & & 14,42 & 22,31 & & 15,42 \\
\hline Cefalosporinas de primeira geração - J01DB & & 48,83 & & & & 124,59 & & & 29,69 & & & 8,87 & & 4,86 \\
\hline
\end{tabular}

UTI: unidade de terapia intensive; UTR: unidade de transplante renal; TMO: transplante de medulla óssea; UC: unidade coronariana; UETDI: unidade especial de tratamento de doenças infecciosas; Ortop: ortopedia; Onco: oncologia; Oft_Otor: oftalmologia e otorrinolaringologia; CM 6º clínica médica do sexto andar; CM 5: clínica médica do quinto andar; GO: ginecologia e obstetrícia; Derm: dermatologia; Neuro: neurologia. Fonte: Autores. 
Ainda em relação ao grupo TE, os carbapenêmicos foram os mais utilizados em três enfermarias [UTI, Neurologia e Unidade de Transplante Renal (UTR)] e as cefalosporinas de $4^{\circ}$ geração foram a classe de antibióticos mais indicada em outras três enfermarias [Transplante de Medula Óssea (TMO), oncologia e clínica médica do $6^{\circ}$ andar $\left(\mathrm{CM} 6^{\circ}\right)$ ]. A enfermaria de ginecologia foi a única unidade onde os carbapenêmicos não se encontram entre os antibióticos que representaram $90 \%$ do consumo. Cabe ressaltar também que as cefalosporinas de $4^{\circ}$ geração e os glicopeptídeos estão entre aqueles que representaram mais de $90 \%$ do consumo em todas as enfermarias

As tetraciclinas apresentam-se entre os antibióticos 90\% mais consumidos na UTI e na enfermaria de dermatologia. Sobre a utilização de antibióticos nas unidades de cuidado de doenças infecciosas [UETDI e moléstias infecciosas (MI)], as sulfonamidas de ação intermediária e a combinação de sulfonamidas com trimetoprima foram as classes de medicamentos mais utilizadas.

A Tabela 2 ilustra o padrão de utilização de antibióticos para TD que correspondem aos $90 \%$ mais consumidos entre as enfermarias. A UTI foi o local de maior utilização de antibióticos para TD, seguido da ortopedia. No grupo TD, antibióticos de amplo espectro também tiveram um consumo elevado. Na UTI, a polimixina foi a classe mais utilizada. Os carbapenêmicos foram os mais utilizados na Unidade Coronariana (UC), UTR e CM $6^{\circ}$. Na oncologia, as cefalosporinas de $4^{\circ}$ geração foram as mais utilizadas e na TMO os carbapenêmicos e as cefalosporina de $4^{\circ}$ geração apresentaram o maior consumo. A TMO foi a enfermaria com a menor taxa de utilização de antibióticos por TD. Houve, portanto uma queda importante de consumo nesta unidade em relação ao grupo TE. Nas unidades de MI e UETDI, os macrolídeos e as fluoroquinolonas foram as classes de antibióticos mais utilizadas para TD. Na enfermaria de oftalmologia e otorrinolaringologia, as penicilinas resistentes à betalactamase se mantiveram como classe mais usada. A ortopedia foi a segunda enfermaria que mais utilizou antimicrobianos para TD, sendo as fluoroquinolonas as mais utilizadas, considerando os grupos TE e TD. As cefalosporinas de $4^{\circ}$ geração e os glicopeptídos deixaram de ser representados em algumas enfermarias quando analisadas para TD. Por outro lado, as penicilinas resistentes à betalactamase passaram a ser representadas em um número maior de enfermarias em relação à TE. Na UTI, esta classe não é representada para TE ao passo que se torna a $3^{\circ}$ mais utilizada no grupo TD. Situação similar pode ser observada na unidade coronariana, UETDI e Neurologia, outros locais onde as penicilinas resistentes à betalactamase são representadas somente para TD. 
Research, Society and Development, v. 11, n. 2, e12011225573, 2022

(CC BY 4.0) | ISSN 2525-3409 | DOI: http://dx.doi.org/10.33448/rsd-v11i2.25573

Tabela 2. Utilização de antibióticos $90 \%$ para tratamento direcionado.

\begin{tabular}{|c|c|c|c|c|c|c|c|c|c|c|c|c|c|c|}
\hline Classe de antióticos/Enfermerais & UTI & UTR & TMO & $U C$ & UETDI & Ortop & Onco. & $M I$ & Oft_Otor & $C M 6^{\circ}$ & $C M 5^{\circ}$ & $G O$ & Derm & Neuro \\
\hline Polimixinas - J01XB & 115,60 & 9,00 & & 14,78 & 24,10 & 13,48 & & & & 4,6 & & 2,8 & 7,1 & \\
\hline Outros aminoglicosídeos - J01GB & 84,21 & & & 23,82 & 5,77 & & & 14,89 & 6,62 & 16,0 & 6,4 & 2,8 & 9,4 & 4,0 \\
\hline Penicilinas resistentes a beta-lactamase - J01CF & 67,82 & 8,93 & & 9,91 & 9,51 & 46,27 & & 23,49 & 31,76 & 27,8 & 42,7 & 5,4 & 51,2 & 3,3 \\
\hline Carbapenêmicos - J01DH & 60,49 & 34,49 & 4,64 & 50,11 & 14,73 & & 12,23 & 17,00 & 4,87 & 32,8 & 15,1 & 4,1 & 10,6 & 5,5 \\
\hline Tetraciclinas - J01AA & 60,40 & & & 21,48 & & 32,30 & & 10,69 & 4,69 & & & & & \\
\hline $\begin{array}{r}\text { Combinações de penicilinas com inibidores de beta- } \\
\text { lactamase - J01CR }\end{array}$ & 42,54 & 25,26 & 3,53 & 31,47 & & 34,16 & 7,75 & 14,85 & 4,95 & 9,5 & 11,2 & 3,0 & 9,3 & 12,1 \\
\hline Fluoroquinolonas - J01MA & 34,26 & 12,03 & & 19,82 & 25,47 & 49,55 & 9,60 & 38,28 & 7,53 & 8,2 & 13,9 & 5,5 & 20,0 & 20,7 \\
\hline Cefalosporinas de terceira geração - J01DD & 33,91 & 6,49 & & 15,56 & 6,12 & 11,20 & & 9,11 & 15,16 & 18,8 & 12,6 & 2,4 & 13,2 & 10,7 \\
\hline Penicilinas de amplo espectro - J01CA & 24,55 & 7,09 & & & 6,05 & 9,86 & 3,56 & 17,09 & 6,95 & & 28,6 & 5,6 & & \\
\hline Glicopeptídeos - J01XA & & & & & & 15,10 & 8,23 & 10,52 & & 10,2 & 9,3 & 1,9 & 21,6 & 9,6 \\
\hline Cefalosporinas de quarta geração - JO1DE & & & 4,64 & & 9,22 & & 14,67 & & 4,24 & 5,3 & & & 6,4 & 4,3 \\
\hline Macrolídeos - J01FA & & & & & 59,14 & & & 16,30 & & & 3,6 & & & \\
\hline Combinações de sulfonamidas e trimetoprim - J01EE & & & & & 12,16 & 16,50 & & 12,02 & & & & & & 5,2 \\
\hline Sulfonamidas de ação intermediária - J01EC & & & & & & & & 9,35 & & & & & & \\
\hline Cefalosporinas de segunda geração - J01DC & & & & & & & & & & & & 7,0 & & \\
\hline
\end{tabular}

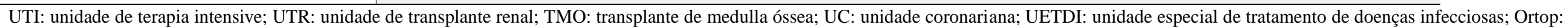

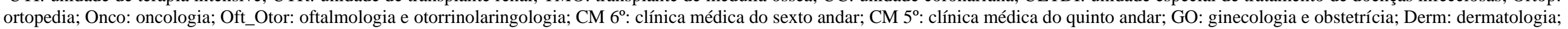
Neuro: neurologia. Fonte: Autores. 
As cinco bactérias mais frequentes nos exames de cultura do grupo TD foram Escherichia coli (19,3\%), Klebsiella pneumoniae (16,0\%), Acinetobacter baumannii (10,9\%), Staphylococcus aureus (10,7\%), Pseudomonas aeruginosa $(9,8 \%)$, correspondendo a $66,7 \%$ de todos os resultados.

Em relação ao perfil de sensibilidade destaca-se que aproximadamente $45 \%$ e $55 \%$ dos resultados com a Escherichia coli são resistentes a cefalosporinas de terceira geração e fluoroquinolonas, respectivamente; somente os aminoglicosídeos, carbapenêmicos, polimixinas e a tigeciclina apresentaram sensibilidade acima de 50\% em Klebsiella pneumoniae; enquanto que em Acinetobacter baumannii, somente a amicacina, a tigeciclina e as polimixinas apresentaram sensibilidade acima de 50\%; e mais de $90 \%$ dos resultados com Staphylococcus aureus foram resistentes para ampicilina e penicilinas sensíveis a penicilinase, e 55,8\% foram resistentes a oxacilina (Figura 1).

Figura 1. Perfil de sensibilidade das bactérias testadas.
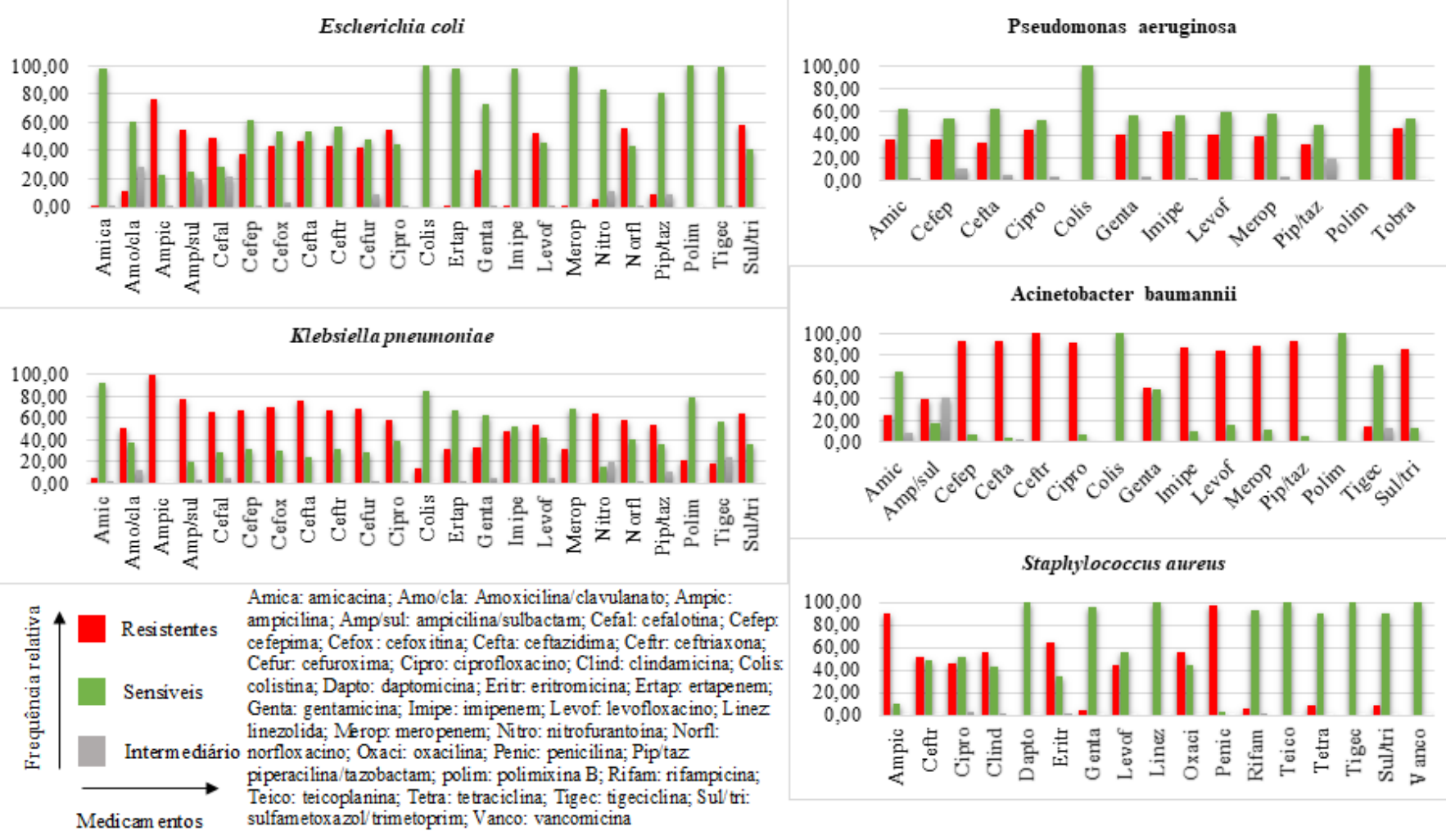

Fonte: Autores.

\section{Discussão}

Os resultados deste estudo são importantes para consolidação dos estudos de utilização de antibióticos no Brasil, visto que a quantidade e a qualidade destes estudos publicados são baixas. Em uma revisão sistemática publicada em 2021, foram encontrados 23 estudos brasileiros, realizados no século XXI, que apresentam resultados de consumo de antibióticos em hospitais. Dois estudos avaliaram o consumo de todos os antibióticos padronizados pelos respectivos hospitais estudados por meio da DDD e sem restringir o local de estudo para UTIs (Pereira et al., 2021).

A análise de utilização de antibióticos mostrou que a UTI é a unidade que apresentou maior consumo geral. Este resultado era esperado e corrobora com achados de outros estudos previamente publicados (Kuster et al., 2008; Stocker et al., 2020), pois a UTI é uma unidade que recebe pacientes em estado crítico de saúde, alguns destes com a imunidade comprometida, e que 
utilizam dispositivos invasivos como cateteres e tubos para ventilação mecânica, oferecendo aos pacientes elevado risco de doenças infecciosas (Vicent et al., 2003).

À vista destas características, a UTI apresentou um padrão de elevado consumo de antibióticos de amplo espectro de ação, como carbapenêmicos, polimixinas e glicopeptídeos. Este padrão é semelhante ao encontrado em outros estudos realizados no Brasil (Pereira et al., 2021), mas diferente quando comparado a outros países. Em estudo realizado em uma unidade de terapia intensiva de um hospital de mesmo porte na Coréia do Sul, observou-se um consumo maior de cefalosporinas de terceira geração (336,10 DDD/1000 pacientes-dia), aminoglicosídeos (219,88 DDD/1000 pacientes-dia) e fluoroquinolonas (210,26 DDD/1000 pacientes-dia) (Kim et al., 2020). Elevado consumo de cefalosporinas de terceira geração (494,59 DDD/1000 pacientes-dia) e fluoroquinolonas (117,13 DDD/ 1000 pacientes-dia) também foi observado em hospital de mesmo porte na Romênia (Zaha et al., 2019).

Ao avaliar o consumo de antibióticos para TD, este padrão modifica-se. Os antibióticos mais utilizados passam a ser polimixinas, aminoglicosídeos e penicilinas resistente a beta-lactamase, enquanto a utilização de glicopeptídeos não aparece mais entre os $90 \%$ mais utilizados. Tais achados mostram um comportamento diferente de ajuste de terapia antimicrobiana quando a bactéria detectada é gram-positiva ou gram-negativa. A proporção maior na utilização de penicilinas resistentes a penicilinase e menor na utilização de glicopeptídeos sugerem o descalonamento de tratamento em consequência à detecção de bactérias gram-positivas sensíveis a oxacilina. Este comportamento é similar na UC, na ortopedia, na $\mathrm{CM} 6^{\circ}$, na $\mathrm{CM} 5^{\circ}$. Ao contrário, o aumento na utilização de polimixinas e aminoglicosídeos indica o provável escalonamento do tratamento a fim de agir contra bactérias gram-negativas multirresistentes detectadas.

Em um estudo multicêntrico realizado em países da América do Sul e do Norte, da Oceania, da Europa e da Ásia, que avaliou o descalonamento de tratamento antimicrobiano em UTIs, encontrou resultados semelhantes ao deste estudo quando o tratamento era direcionado para infecções por bactérias gram-positivas. Os antibióticos mais utilizados empiricamente foram as penicilinas associadas a inibidores de beta-lactamase, carbapenêmicos, cefalosporinas de terceira geração e glicopeptídeos; e os antibióticos mais frequentemente descontinuados foram os glicopeptídeos. No entanto, em relação ao tratamento de bactérias gram-negativas, o estudo apresentou redução na utilização de antibióticos de amplo espectro de ação (com ação antipseudomonas) e aumento na utilização de cefaloporinas de terceira geração. Além disso, o descalonamento foi utilizado com uma frequência muito baixa (16\%), como observado no presente trabalho (Bus et al., 2020).

Além destes achados, Bus et al. 2020, mostraram que o ato de descalonar o tratamento está associado à gravidade da condição clínica do paciente: o descalonamento tende a ocorrer com mais frequência em pacientes menos graves. Este comportamento não foi encontrado neste estudo, visto que não se observou redução no consumo de antibióticos de amplo espectro de ação e aumento no consumo de antibióticos de curto espectro de ação nas enfermarias em que os pacientes não são críticos.

A frequência reduzida na realização de descalonamento do tratamento de infecções causadas por bactérias gramnegativas pode ser explicada pelos resultados do antibiograma. Entre as cinco bactérias que representam 66,7\% das infecções, quatro são gram-negativas e com baixa sensibilidade a maioria dos beta-lactâmicos. No entanto, $79,4 \%$ dos pacientes não tiveram seus tratamentos guiados pelo antibiograma, ou seja, talvez a frequência de infecções causadas por bactérias sensíveis aos antibióticos disponíveis para utilização no hospital seja maior que as presentes nos antibiogramas. Além disso, a média do tempo de internação do grupo TD foi maior do que o tempo de internação do grupo TE, o que permite levantar a hipótese de que o antibiograma tenha sido realizado a fim de fornecer opões terapêuticas ao tratamento que não obteve sucesso.

Alshareef et al. (2020) mostraram que o tempo de internação em pacientes que tiveram descalonamento no tratamento com antibiótico foi significativamente menor quando comparado ao grupo de pacientes que não tiveram o tratamento descalonado. No entanto, todos os pacientes foram avaliados quanto à sensibilidade do agente etiológico da doença infecciosa 
Research, Society and Development, v. 11, n. 2, e12011225573, 2022

(CC BY 4.0) | ISSN 2525-3409 | DOI: http://dx.doi.org/10.33448/rsd-v11i2.25573

acometida, e o não descalonamento do tratamento associou-se a resultados positivos de bactérias multirresistentes, o que reforça a hipótese levantada anteriormente. O contrário foi observado em um ensaio clínico randomizado, que mostrou que o tempo de internação de pacientes diagnosticados com sepse grave foi significativamente maior quando houve o descalonamento do tratamento comparado ao grupo de pacientes que mantiveram a TE (Leone et al., 2014).

A TMO foi a enfermaria com o menor número de antibióticos entre os $90 \%$ mais utilizados quando comparado às outras enfermarias tanto no grupo TE quanto no TD. Os antibióticos mais utilizados foram as cefalosporinas de quarta geração e os carbapenêmicos. Este perfil de utilização de antibióticos está associado ao tratamento de neutropenia febril, cuja incidência é maior que $80 \%$ em pacientes com neoplasia hematológica maligna (Schmidt-Hieber et al., 2019). Portanto este consumo de antibióticos era esperado, visto que o protocolo do HCFMRP preconiza a utilização empírica de antibióticos bactericidas com atividade anti-pseudomonas, com preferência à cefepima e restringindo a utilização dos carbapenêmicos para falha terapêutica. Em um estudo realizado utilizando uma base nacional de dados hospitalares da Austrália mostrou que a principal indicação de uso de antibióticos na TMO foi para o tratamento de neutropenia febril, no entanto o antibiótico mais prescrito foi a piperacilina-tazobactam (48\% das prescrições), seguido do meropenem (15\%) e vancomicina (12\%) (Douglas et al., 2021). Embora estes dois estudos apresentem diferenças no antibiótico mais utilizado para o tratamento de neutropenia febril, ambos estão de acordo com as recomendações internacionais de tratamento desta condição (antibióticos com atividade anti-pseudomonas de baixa toxicidade) (Freifeld et al., 2011).

Sulfonamidas de ação intermediária, combinação de sulfonamidas com trimetoprim, macrolídeos e cefalosporinas de terceira geração foram as classes mais utilizadas para TE na UETDI e MI. Isso tem provável relação com o fato de serem indicadas ao tratamento de infecções oportunistas, como, sulfametoxazol + trimetoprim no tratamento de toxoplasmose e macrolídeos e cefalosporinas de terceira geração no tratamento de pneumonia bacteriana, em pacientes imunodeprimidos (Feldman et al. 2016; Pellegrino et al.2019). O consumo reduzido destas mesmas classes no grupo TD pode ser explicado pela ausência de efetividade em bactérias resistentes, evidência fortalecida pelo aumento no consumo de fluoroquinolonas e polimixina.

O método descritivo com desenho transversal e a análise de dados agregados realizado neste estudo limita a verificação das causas que levaram ao padrão de utilização de antibióticos encontrados neste estudo. Além disso, a coleta de dados em fontes secundárias impede que o pesquisador tenha um controle maior das informações coletadas, como as variáveis de confusão.

Apesar disso, estudos de utilização de antibióticos utilizando as métricas propostas pelo OMS são incipientes no Brasil, como observado na revisão sistemática realizada por Pereira et al. 2021. Visto que este tipo de estudo permite o levantamento de hipóteses que podem ser testadas futuramente, este é o primeiro passo para um entendimento do padrão de utilização de antibióticos realizado no Brasil, e assim pode contribuir significativamente para a elaboração de estratégias e programas de uso racional de antibióticos desde o nível hospitalar local até o nível nacional.

\section{Conclusão}

O consumo de antibióticos de amplo espectro de ação foi elevado com pequenas variações entre as enfermarias estudadas, representadas pelas particularidades de cada especialidade médica. O baixo consumo de antibióticos para tratamento direcionado, somado a semelhança no padrão de consumo de antibióticos de amplo espectro de ação, a elevada frequência de bactérias gram-negativas resistentes, e o elevado tempo de internação no grupo TD sugere a hipótese de que os exames de antibiograma estão sendo realizados com o objetivo de investigar o perfil de sensibilidade bacteriana quando há falha terapêutica, e não para direcionar o tratamento. Sendo assim, a ampliação na realização de antibiogramas para o 
direcionamento do tratamento antimicrobiano seria uma estratégia importante para melhorar o consumo de antibióticos. Além disso, é importante que estudos analíticos longitudinais sejam realizadas para confirmar esta hipótese apresentada.

\section{Agradecimentos}

Agradecimento ao apoio financeiro da Coordenação de Aperfeiçoamento de Pessoal de Nível Superior (CAPES) Brasil, código de financiamento 001.

\section{Referências}

Alshareef, H.; Alfahad, W.; Albaadani, A.; Alyazid, H.; \& Talib, R. B. (2020). Impact of antibiotic de-escalation on hospitalized patients with urinary tract infections: A retrospective cohort single center study. J Infect Public Health, 13, 985-990.

Benko, R.; Matuz, M.; Doro, P.; Viola, R.; Hajdu, E.; Monnet, D. L.; et al. (2009). Hungarian Hospital Antibiotic Consumption at the Regional Level, 19962005. Infection, 37 (2), 133-137.

Bergman, U.; Popa, C.; Tomson, Y.; Wettermark, B.; Einarson, T. R.; Aberg, H.; et al. (1998). Drug utilization 90\% - a simple method for assessing the quality of drug prescribing. Eur J Clin Pharmacol, 54, 113-118.

Bus, L.; Depuydt, P.; Steen, J.; Dhaese, S.; Smet, K.; Tabah, A.; et al. (2020). Antimicrobial de-escalation in the critically ill patients and assessment of clinical cure: the DIANA study. Intensive Care Med, 46, 1404-1417.

Castro, C. G. S. O (2002). Estudos de utilização de medicamentos: noções básicas, Editora Fiocruz.

Cheng, A. C.; Turnidge, J.; Collignon, P.; Looke, D.; Barton, M.; \& Gottlieb, T. (2012) Control of Fluoroquinolone Resistance through Succesful Regulation, Australia. Emerg Infect Dis, 18 (9), 1453-1460.

Douglas, A. P.; Hall, L.; James, R. S.; Worth, L. J.; Slavin, M. A.; \& Thursky, K. A. (2021). Quality of inpatients antimicrobial use in hematology and oncology patients. Infect Control Hosp Epidemiol, 42 (10), 1235-1244.

Feldman, C.; \& Anderson, R. (2016). Bacterial Respiratory Infections Complicating Human Immunodeficiency Virus. Semin Respir Crit Care Med, 37, 214229.

Freifeld, A. G.; Bow, E. J.; Sepkowitz, K. A.; Boeckh, M. J.; Ito, J. I.; Mullen, C. A.; et al. (2011). Clinical Practice Guideline for the Use of Antimicrobial Agents in Neutropenic Patients with Cancer: 2010 Update by the Infectious Diseases Society of America. Clin Infect Dis, 52 (4), e56-e93.

Kim, B.; Hwang, H.; Kim, J.; Lee, M.; \& Pai, H. (2020). Tem-year trends in antibiotic usage at a terciary care hospital in Korea, 2004 to 2013. Korean J Intern Med, 35, 703-713.

Kuster, S. P.; Ruef, C.; Bollinger, A. K.; Ledergerber, B.; Hintermann, A.; Deplazes, C.; et al. (2008). Correlation betweem case mix index and antibiotics use in hospitals. J Antimicrob Chemother, 62, 837-842.

Leone, M.; Bechis, C.; Baumstarck, K.; Lefrant, J.; Albanèse, J.; Jaber, S.; et al. (2014). De-escalation versus continuation of empirical antimicrobial treatment in severe sepsis: a multicenter non-blinded randomized noninferiority trial. Intensive Care Med, 40, 1399-1408.

Medronho, R. A.; Bloch, K. V.; Luiz, R. R.; Werneck, G. L.; et al. (2008). Epidemiologia. Atheneu.

Pellegrino, D.; Gryschek, R.; Oliveira, A. C. P.; Marcusso, R.; Correia, A.; \& Vidal, J. E. (2019). Efficacy and safety of trimethoprim-sulfamethoxazole in HIV-infected patients with cereberal toxoplasmosis in Brazil: a single-arm open-label clinical trial. Int J STD AIDS, 30 (12), $1156-1162$.

Pereira, L. B.; Zanetti, M. O. B.; Sponchiado, L. P.; Rodrigues, J. P. V.; Campos, M. S. A. C.; Varallo, F. R.; et al. (2021). Antibiotic use in Brazilian hospitals in the 21st century: a systematic review. Rev Soc Bras Med Trop, 54, e0861.

Schmidt-Hieber, M.; Teschner, D.; Maschmeyer, G.; \& Schalk, E. (2019). Management of febrile neutropenia in special consideration of the role of antimicrobial de-escalation. Expert Rev Anti Infect Ther, 17 (12), 983-995.

Stocker, H.; Mehlhorn, C; Jordan, K.; Eckholt, L.; Jefferys, L.; \& Arastéh, K. (2020). Clinical and economic effects of an antimicrobial stewardship intervention in a surgical intensive care unit. Infection, 48, 509-519.

Vicent, J. L.; Rello, J.; Marshall, J.; Silva, E.; Anzueto, A.; Martin, C. D.; et al. (2009). International Study of the Prevalence and Outcomes of Infection in Intensive Care Units. JAMA, 302 (21), 2323-2329.

WHO. World Health Organization. (2014). International Classification of Diseases 10th Revision. ICD-10. https://icd.who.int/browse10/2015/en.

WHO. World Health Organization. (2015). ATC/DDD Index 2015. https://www.whocc.no/atc_ddd_index/

Zaha, D. C.; Bungau, S.; Aleya, S.; Tit, D. M.; Vesa, C. M.; Popa, A. R.; et al. (2019). What antibiotics for what pathogens? The sensitivity spectrum of isolated strains in an intensive care unit. The Science of the total environment, 687, 118-127. 